\title{
ON MORSE THEORY AND STATIONARY STATES FOR NONLINEAR WAVE EQUATIONS
}

\author{
BY MELVYN S. BERGER ${ }^{1}$
}

Communicated by J. T. Schwartz, December 1, 1969

The nonlinear equations to be discussed here can be written in the form

$$
\begin{aligned}
& \partial^{2} u / \partial t^{2}=L u+N(x, u), \\
& i \partial u / \partial t=L u+N(x, u)
\end{aligned}
$$

where $L$ is a second order elliptic formally selfadjoint differential operator acting on complex-valued functions $u(t, x)$ defined on $\boldsymbol{R}^{1} \times \boldsymbol{R}^{3}$, and $N(x, u)=f\left(x,|u|^{2}\right) u$ is a complex-valued function jointly continuous in $x$ and $u$ with $f(x, r)=o(1)$ as $|r| \rightarrow \infty$. A complexvalued function $u(t, x)$ is called a stationary state of (1) [or $\left(1^{\prime}\right)$ ] if

(a) $u(t, x)$ satisfies (1) [or $\left(1^{\prime}\right)$ ] on $R^{1} \times R^{3}$, and

(b) $u(t, x)=v(x) e^{i \lambda t}$ where $\lambda$ is some real number and $v(x)$ is a smooth real-valued function defined on $R^{3}$, tending to 0 exponentially as $|x| \rightarrow \infty$ but not identically zero.

In this article we wish to examine the structure and properties of the stationary states of $(1)$ [or $\left(1^{\prime}\right)$ ] by combining recent results of Morse theory on Hilbert manifolds with concrete estimates for elliptic differential operators defined on $R^{3}$.

1. Statement of basic results. We begin with two affirmative facts concerning the existence of stationary states.

THEOREM 1. Let $L=\Delta-p^{2}$ ( $p=$ const.), $f(x, u)=k(|x|)|u|^{\sigma}$ with $0<\sigma<4$ where $k(|x|)$ is a bounded positive continuous function uniformly bounded above zero. Then (1) and (1') have (for each $\lambda^{2}<p^{2}$ in (1) and $\lambda<p^{2}$ in (1')) a countably infinite number of stationary states $v_{N}(x, \lambda), N=0,1,2, \cdots$ Each $v_{N}(x, \lambda)$ has precisely $N$ nodal domains in $R^{3}$ and is nonoscillatory outside some fixed sphere of radius $R$ (independent of $N$ ).

THEOREM 2. The $v_{N}(x, \lambda)$ of Theorem 1 (apart from a constant multiplier) are limits (as $m \rightarrow \infty$ ) of spherically symmetric nondegenerate critical points $v_{N m}(x, \lambda)$ of index $N$ of the functional $\int_{B_{m}} k(|x|)|u|^{\sigma} u^{2}$ on an infinite dimensional Hilbert manifold $\mathfrak{N}_{m}$ where $B_{m}$ is a ball

$A M S$ Subject Classifications. Primary 5815; Secondary 3560.

Key Words and Phrases. Morse theory, nonlinear wave equations.

1 Research partially supported by NSF grant 16578. 
of radius $m$ in $R^{3}$ centered at the origin. $\mathfrak{T}_{m}$ is obtained by intersecting real projective space $P^{\infty}(H)$ (obtained by identifying the antipodal points of the sphere

$$
\int_{B_{m}}|\nabla u|^{2}+\left|C_{\lambda}\right| u^{2}=1, \quad C_{\lambda}=\text { const., }
$$

of the Hilbert space $\left.H=\dot{W}_{1,2}\left(B_{m}\right)\right)$ with spherically symmetric functions of the form $u(x)=v(r) / r$ where $v(0)=0$ and $v(|x|) \in \dot{W}_{1,2}\left(B_{m}\right)$.

On the other hand, we have the following results concerning the nonexistence of stationary states.

THEOREM 3. Let $L u=\Delta u-p(x) u$ where $p(x)=p^{2}+o\left(|x|^{-1}\right)$ is a nonnegative function continuous outside some bounded domain. Then neither (1) nor ( $\left.1^{\prime}\right)$ possesses stationary states for any $\lambda \geqq p^{2}$ in $\left(1^{\prime}\right)$ or $\lambda^{2} \geqq p^{2}$ in (1).

THEOREM 4. In addition to the hypotheses of Theorem 3, suppose $f(x, u)=|u| \sigma$. Then if $\sigma \geqq 4$, neither (1) nor $\left(1^{\prime}\right)$ possesses stationary states for any $\lambda$.

2. Sketch of proofs. The results (Theorems 3 and 4) concerning the nonexistence of stationary states follow immediately from Kato [1] and the following "invariant integral" for solutions of

$$
\Delta v+\partial F(v) / \partial v=0
$$

defined on $R^{3}$ and vanishing sufficiently rapidly at $\infty$

$$
6 \int_{R^{z}} F(v) d x=\int_{R^{s}} v \frac{\partial F(v)}{\partial v} d x .
$$

Theorem 1 follows by noting that the stationary states of $\left(1^{\prime}\right)$ can be obtained (after scaling) as the critical points of the functional $\int_{R^{v}} k(x)|v| \sigma_{v^{2}}$ on the class of functions

$$
U=\left\{\left.v\left|\int_{R^{3}}\right| \nabla v\right|^{2}+\left|p^{2}-\lambda\right| v^{2}=1, v \in \dot{W}_{1,2}\left(R^{3}\right)\right\} .
$$

These critical points can, in turn, be approximated by replacing $R^{3}$ by the ball $B_{m}$ of sufficiently large radius $m$. (The argument is similar for (1).) In addition we restrict attention to critical points $C_{m}$ of the form $v(x)=w(|x|) /|x|$ where $w(r) \in \dot{W}_{1,2}(0, m)$. We then apply the Morse theory of critical points of the functional

$$
G(w)=\int_{0}^{m} k(r) r^{1-\sigma}(w(r))^{\sigma+1} d r
$$


on the infinite dimensional projective space $\widetilde{P}^{\infty}$ defined by identifying antipodal points of the set

$$
\left\{w\left|F(w)=\int_{0}^{m} \dot{w}^{2}+\right| p^{2}-\lambda \mid w^{2}=1, w \in \dot{W}_{1,2}(0, m)\right\} .
$$

For a suitable sequence $m_{n} \rightarrow \infty$, these critical points are all nondegenerate and the variational problem satisfies the compactness condition: any sequence $w_{n} \in \widetilde{P}^{\infty}$ with $\left\{\operatorname{grad} F\left(w_{n}\right)\right\}$ weakly convergent implies $\left\{\operatorname{grad} G\left(w_{n}\right)\right\}$ is strongly convergent (and hence the Palais-Smale condition $C$ for $1 / G(w))$. Applying the results of $\mathrm{J}$. Schwartz [2] to this problem, we note $G(w)$ has a sequence of critical points $w_{N m}(N=0,1,2, \cdots)$ of index $N$ such that $G\left(w_{N m}\right)>G\left(w_{N+1, m}\right)$ for all $N$. This fact together with the classical oscillation theory for second order ordinary differential equations implies

(i) $w_{N m}$ has precisely $N$ nodal domains in $B_{m}$, and

(ii) that the number of these nodal domains does not change when $m \rightarrow \infty$.

An important point in the above arguments (especially in showing that $\lim _{m \rightarrow \infty} w_{N m}=w_{N}$ is a stationary state) is the following a priori bound for critical points $C_{m}$.

LEMmA. For $m \geqq M_{0}$ sufficiently large and for fixed $\lambda$, any critical point $v \in C_{m}$ satisfies

$$
|v(x)| \leqq C\left(e^{-\beta|x|} /|x|\right), \quad \text { for }|x| \geqq A, \quad \beta^{2}=\left(p^{2}-\lambda\right),
$$

where $C$ and $A$ are constants independent of $m$.

\section{BIBLIOGRAPHY}

1. T. Kato, Growth properties of solutions of the reduced wave equation with a variable coefficient, Comm. Pure Appl. Math. 12 (1959), 403-425. MR 21 \#7349.

2. J. Schwartz, A remark on Morse theory, Comm. Pure Appl. Math. 19 (1966), 139-143. MR 34 \#827.

Belfer Graduate School of Science, Yeshiva University, New York, NEW YoRK 10033 